\title{
Stem-Loop RT-qPCR as an Efficient Tool for the Detection and Quantification of Small RNAs in Giardia lamblia
}

\author{
Jaime Marcial-Quino ${ }^{1, *, \dagger}$, Saúl Gómez-Manzo ${ }^{2, \dagger}$, Francisco Fierro ${ }^{3}$, America Vanoye-Carlo ${ }^{4}$, \\ Yadira Rufino-González ${ }^{5}$, Edgar Sierra-Palacios ${ }^{6}$, Adriana Castillo-Villanueva ${ }^{2}$, \\ Rosa Angélica Castillo-Rodríguez ${ }^{1}$, Eduardo Rodríguez-Bustamante ${ }^{7}$, \\ Roberto Arreguin-Espinosa ${ }^{7}$ and Horacio Reyes-Vivas ${ }^{2}$
}

1 CONACYT-Instituto Nacional de Pediatría, Secretaría de Salud, Ciudad de México 04530, Mexico; racastilloro@conacyt.mx

2 Laboratorio de Bioquímica Genética, Instituto Nacional de Pediatría, Secretaría de Salud, Ciudad de México 04530, Mexico; saulmanzo@ciencias.unam.mx (S.G.-M.); acastilloinp@gmail.com (A.C.-V.); hreyesvivas@yahoo.com.mx (H.R.-V.)

3 Laboratorio de Ingeniería Genética y Metabolitos Secundarios, Departamento de Biotecnología, Universidad Autónoma Metropolitana-Iztapalapa, Ciudad de México 09340, Mexico; degfff@yahoo.com

4 Laboratorio de Neurociencias, Instituto Nacional de Pediatría, Secretaría de Salud, Ciudad de México 04530, Mexico; america_vc@yahoo.com.mx

5 Laboratorio de Parasitología Experimental, Instituto Nacional de Pediatría, Secretaría de Salud, Ciudad de México 04530, Mexico; yadirg@gmail.com

6 Colegio de Ciencias y Humanidades, Plantel Casa Libertad, Universidad Autónoma de la Ciudad de México, Ciudad de México 09620, Mexico; edgar.sierra@uacm.edu.mx

7 Departamento de Química de Biomacromoléculas, Instituto de Química, Universidad Nacional Autónoma de México, Circuito Exterior s/n, Ciudad Universitaria, Ciudad de México 04510, Mexico;

e-rodriguez-bustamante@ciencias.unam.mx (E.R.-B.); arrespin@unam.mx (R.A.-E.)

* Correspondence: jmarcialqu@conacyt.mx; Tel.: +52-55-1084-0900 (ext. 1442); Fax: +52-55-1084-3883

+ These authors contributed equally to this work.

Academic Editor: Paolo Cinelli

Received: 3 September 2016; Accepted: 26 November 2016; Published: 20 December 2016

\begin{abstract}
Stem-loop quantitative reverse transcription PCR (RT-qPCR) is a molecular technique used for identification and quantification of individual small RNAs in cells. In this work, we used a Universal ProbeLibrary (UPL)-based design to detect-in a rapid, sensitive, specific, and reproducible way-the small nucleolar RNA (snoRNA) GlsR17 and its derived miRNA (miR2) of Giardia lamblia using a stem-loop RT-qPCR approach. Both small RNAs could be isolated from both total RNA and small RNA samples. Identification of the two small RNAs was carried out by sequencing the PCR-amplified small RNA products upon ligation into the pJET1.2/blunt vector. GlsR17 is constitutively expressed during the $72 \mathrm{~h}$ cultures of trophozoites, while the mature miR2 is present in 2 -fold higher abundance during the first $48 \mathrm{~h}$ than at $72 \mathrm{~h}$. Because it has been suggested that miRNAs in G. lamblia have an important role in the regulation of gene expression, the use of the stem-loop RT-qPCR method could be valuable for the study of miRNAs of G. lamblia. This methodology will be a powerful tool for studying gene regulation in G. lamblia, and will help to better understand the features and functions of these regulatory molecules and how they work within the RNA interference (RNAi) pathway in G. lamblia.
\end{abstract}

Keywords: Giardia lamblia; stem-loop RT-qPCR; small nucleolar RNA; microRNA 


\section{Introduction}

Giardia lamblia is a flagellate protozoan that adapted over time to a parasitic lifestyle [1]. This organism causes the disease known as giardiasis in humans, but can also affect several vertebrate organisms [2]. Since G. lamblia is an early divergent microorganism, it has been taken as a good model to study gene regulation, because it shares mechanisms with both prokaryotic and eukaryotic organisms [3].

It is known that small molecules of RNA regulate important cellular processes in different eukaryotic organisms, including G. lamblia [4]. Small nucleolar RNA (snoRNA) has been identified as a source of microRNAs (miRNAs) in some protozoans and metazoans [5-10]. These miRNAs are small RNA molecules that regulate gene expression in lower and higher eukaryotes, but they have not been studied in detail in Giardia [11], although the origins and evolution of miRNAs and other regulatory small RNAs have been extensively characterized [12]. Some miRNAs (miR1, miR2, miR3, miR6, miR10) derived from snoRNA have been identified in G. lamblia by small RNA isolation and ligation to RNA linkers [6], and also by sequencing analysis followed by primer extension [7]. The biogenesis of the different classes of small RNAs shows important differences, as has been previously reported [5,13-16]. At least 166 miRNAs have been reported in G. lamblia founded by in silico prediction, analysis of homology searching, or deep sequencing $[4,6,7,17,18]$. Studies on the functionality of miRNAs indicated that they may have an important role on the regulation of genes encoding variant-specific surface proteins (VSPs) [6,7]; each of these miRNAs showed specific binding sites in the sequence of VSP genes [7,17,18].

Recently, it was reported that VSP genes are transcribed simultaneously in sense and antisense strands, which was proposed to result in the formation of double-stranded RNAs (dsRNAs) that are then degraded by the Dicer endonuclease complex [19]. Long dsRNAs can serve as templates for the biogenesis of small RNAs; this form of post-transcriptional gene silencing (PTGS) is a mechanism similar to the RNA interference (RNAi) pathway [20-22]. Other molecules, such as endo-small interfering RNAs (endo-siRNAs) and tRNAs derived from small RNAs, have been shown to play a key role in the differentiation of Giardia, suggesting that they might be involved in the regulation of this process [23]. These studies confirm that G. lamblia possesses expression regulatory mechanisms by PTGS or through the RNAi pathway involving small RNAs [6,18,21-23]. Moreover, several elements of the RNAi silencing pathway have been identified in Giardia, making conceivable its existence and usefulness to perform specific gene silencing. Analysis of the Giardia genome has revealed the presence of one copy each of the Dicer, Argonaute $(A g o)$, and RNA-dependent RNA polymerase $(R d R p)$ genes $[6,21,24]$. Although Drosha and Exportin 5 homologs are absent in this parasite, there is the possibility of another Drosha-independent pathway involved in miRNA biogenesis from snoRNA $[4,6]$.

Because of the importance of small RNAs, different techniques have been used to detect miRNAs, including isolation of small RNA and ligation to RNA linkers (which requires the presence of a 5'-phosphate in the small RNA) [25], Northern blot [26,27], primer extension [28], microarrays [29], and real-time PCR [30]. In G. lamblia, fluorescence in situ hybridization (FISH) was used to find the cellular location of the snoRNA GlsR17 and its product, miR2 [6]; however, these techniques require time, and they can be laborious, expensive, and have low sensitivity [27]. Stem-loop quantitative reverse transcription PCR (RT-qPCR) is an efficient and novel strategy that has been used in recent years to detect and amplify mature miRNAs [31-34]. The stem-loop is designed such that it is shaped as a hairpin and possesses a $3^{\prime}$ overhang complementary to the miRNA. Also, a miRNA-specific forward primers with a $5^{\prime}$ adapter and a universal reverse primer are design for PCR amplification; these features allow a high specificity and increased flexibility in the design of primers for identification of microRNAs [35,36].

In this paper, we describe the use of the stem-loop RT-qPCR technique to detect different small RNAs in G. lamblia. With this technique, we could isolate, identify, and quantify the expression of GlsR17 (ID: AY820298.1) and its derived mature miRNA, miR2. As far as we know, this is the first report that demonstrates the use of the stem-loop RT-qPCR method for the identification and validation 
of small RNAs in this parasite. This technique can thus be adopted for the quantification of small RNAs in G. lamblia, and used in analyses of genes that are regulated by small RNAs through the RNAi pathway.

\section{Materials and Methods}

\subsection{Strains and Experimental Conditions}

The WB strain of G. lamblia was obtained from the American Type Culture Collection (ATCC). Trophozoites were grown in tubes containing $7 \mathrm{~mL}$ of TYI-S-33 medium (pH 7.02) supplemented with $10 \%$ fetal bovine serum and antibiotics (ampicillin, cephalothin, amphotericin, 10, 10 and $5 \mu \mathrm{g} / \mathrm{mL}$, respectively) and incubated at $37^{\circ} \mathrm{C}$. Upon reaching a confluent monolayer (after approximately $60 \mathrm{~h}$ ) the cells were placed on ice for $20 \mathrm{~min}$ and then collected by centrifugation at $3400 \times g$; the medium was discarded completely and the cells were washed twice with phosphate saline buffer before RNA extraction.

\subsection{RNA Samples: Total RNA and Small RNA}

Total RNA was extracted using TRIzol ${ }^{\circledR}$ Reagent (Invitrogen, Carlsbad, CA, USA) according to the manufacturer's instructions. The concentration and the purity of the isolated RNA were quantified using a NanoDrop ND-1000 Micro-Volume (NanoDrop Technologies, Wilmington, DE, USA), and its integrity was verified in a $0.8 \%(w / v)$ agarose gel under denaturing conditions.

The extraction of small RNAs was performed with the mirPremier ${ }^{\circledR}$ microRNA Isolation Kit (Sigma-Aldrich, St. Louis, MO, USA), following the instructions of the manufacturer's protocol. The small RNA obtained was electrophoresed in 3\% $(w / v)$ agarose gels and visualized with GelRed (Nucleic Acid Gel, Biotium; Hayward, CA, USA) on a MultiDoc-It (UVP; Upland, CA, USA).

In addition, RNAs with sizes $\approx 100$ bp were copurified from total RNA and obtained by the aforementioned kit, following the protocol of Malone et al. [37] and Nielsen [38], with some modifications for this work.

\subsection{Primers and Endpoint $P C R$}

The sequence for primer design of the GlsR17-Fw primer was obtained from GenBank, Accession ID: AY820298.1, and then compared to the reported sequence in the Giardia genome database (http://giardiadb.org/giardiadb/) [39]. The primers used in this study are shown in Table 1.

Table 1. Primers used in this study.

\begin{tabular}{ll}
\hline Primer & Sequence $\left(\mathbf{5}^{\prime}-\mathbf{3}^{\prime}\right)$ \\
\hline Stem-loop-GlsR17 & GTTGGCTCTGGTGCAGGGTCCGAGGTATTCGCACCAGAGCCAACGACTAT \\
GlsR17-Forward & TGTTTTGTTCTAGACCTCCTGG \\
qGlsR17-Forward & CAGGACACAGGCGGAG \\
miR2-Forward & TGCAGCCTAATCACCGC \\
UniLoop-Reverse & GTGCAGGGTCCGAGGT \\
pJET1.2 Forward & CGACTCACTATAGGGAGAGCGGC \\
pJET1.2 Reverse & AAGAACATCGATTTCCATGGCAG \\
pJET2-Forward & CAATTAGTAGCATCACGC \\
Ald-Forward & GAGTCCGTGAAGATGGCGA \\
Ald-Reverse & GTCCCAAGTTCAGCCTCCAC \\
\hline
\end{tabular}

Primers were tested by endpoint PCR to analyze their specificity using CDNA as a template (see next section) and the enzyme Phusion High-Fidelity DNA polymerase (Thermo Scientific, Carlsbad, CA, USA), with the following amplification conditions: $30 \mathrm{~s}$ at $98^{\circ} \mathrm{C} ; 30$ cycles of $10 \mathrm{~s}$ at $98^{\circ} \mathrm{C}, 15 \mathrm{~s}$ at $60{ }^{\circ} \mathrm{C}$, and $15 \mathrm{~s}$ at $72{ }^{\circ} \mathrm{C}$; and, finally, $2 \mathrm{~min}$ at $72{ }^{\circ} \mathrm{C}$. After amplification, the PCR products were separated by electrophoresis on a $3 \%(w / v)$ agarose gel. PCR products were also resolved by electrophoresis through $16 \%$ polyacrylamide gels to improve the resolution of the bands; these gels 
were performed according to the method reported by other authors [40], with some modifications. The gels were stained with GelRed (Nucleic Acid Gel, Biotium) and visualized on a MultiDoc-It (UVP). Synthesis of primers and sequence analysis were carried out at the Unidad de síntesis y secuenciación del Instituto de Biotecnología, UNAM (Cuernavaca, Mexico).

The PCR products were purified and ligated into the pJET1.2/blunt vector (CloneJET PCR Cloning Kit; Thermo Scientific). Each construction was transformed into competent Escherichia coli TOP10F' cells (Invitrogen), which were grown at $37^{\circ} \mathrm{C}$ overnight on Luria-Bertani (LB) agar plates supplemented with $100 \mu \mathrm{g} / \mathrm{mL}$ ampicillin. The plasmidic DNA was extracted by using the GeneJET Plasmid Miniprep Kit (Thermo Scientific), as indicated by the manufacturer, and then the cloned fragments were sequenced.

\subsection{Stem-Loop RT-qPCR Assay Design}

We are proposing a stem-loop RT-qPCR approach for the identification and quantification of small RNAs (snoRNA/miRNA) in G. lamblia. This methodology consists of two steps: reverse transcription (RT) and real-time PCR. First, the stem-loop probe Stem-loop-GlsR17 was used to specifically capture the snoRNA GlsR17 and perform reverse transcription with the Revertaid reverse transcriptase (Thermo Scientific). Then, the RT product was quantified by RT-qPCR using SYBR Green (Figure 1A). The Stem-loop-GlsR17 Universal ProbeLibrary (UPL) probe, the GlsR17- and miR2-specific primers, and the universal reverse primer (UniLoop) were all designed using the UPL probe-based stem-loop quantitative PCR assay design software (http:/ /genomics.dote.hu:8080/ mirnadesigntool) [32]. This software is open source under the GNU General Public License, and access to the source code can be requested by contacting the corresponding author of this software.

\subsection{Synthesis of First-Strand cDNA}

For complementary DNA (cDNA) synthesis, $1 \mu \mathrm{g}$ of total RNA was first treated with the enzyme DNase I (Thermo Scientific) to remove DNA contamination. Complementary DNA was synthesized from DNase I-treated total RNA or from small RNA, using the Stem-loop-GlsR17 primer in $20 \mu \mathrm{L}$ reaction volume with dNTP mix $(10 \mathrm{mM})$ and Revertaid reverse transcriptase (Thermo Scientific). The reaction was incubated for $30 \mathrm{~min}$ at $16^{\circ} \mathrm{C}$ followed by $60 \mathrm{~min}$ at $42^{\circ} \mathrm{C}$, and then stopped by heating at $70{ }^{\circ} \mathrm{C}$ for $10 \mathrm{~min}$. The cDNA was stored at $-20^{\circ} \mathrm{C}$ until use.

\section{6. $R T-q P C R$ Assay}

Amplification was carried out in a StepOne ${ }^{\mathrm{TM}}$ Real-Time PCR System (Applied Biosystems, Foster City, CA, USA). The reactions were performed with the Fast SYBR ${ }^{\circledR}$ Green Master Mix kit (Applied Biosystems), using the following conditions: $95^{\circ} \mathrm{C}$ for $30 \mathrm{~s}$, followed by 40 cycles of $95{ }^{\circ} \mathrm{C}$ for $3 \mathrm{~s}$ and $60^{\circ} \mathrm{C}$ for $30 \mathrm{~s}$. Upon completion of the reaction cycles, melt curves were obtained by heating the reactions from $60{ }^{\circ} \mathrm{C}$ to $95^{\circ} \mathrm{C}$. The specificity of the primers was confirmed by the presence of a single peak in the melt curve generated for GlsR17 and miR2. Negative controls were a reaction lacking the reactive and a reaction without template (NTC control) to verify the absence of contaminating DNA. Calculation of the PCR amplification efficiency for each primer was performed using 10-fold dilution series of cDNA as a template and applying the formula $\mathrm{E}=10^{(-1 / \mathrm{k})}$ [41-43], where $\mathrm{k}$ is the slope. The slope value was obtained from the standard curve generated by the StepOne software v2.3 (Applied Biosystems). Normalization was performed using the geometric mean values of the cycle thresholds (Ct). Finally, the $2^{-\Delta \Delta \mathrm{Ct}}$ method was used for the quantification of the relative expression of the small RNAs of interest [44]. The expression level of the aldolase (Ald) housekeeping gene was employed for equal loading control in all experiments [45]. Five replicates were made of each reaction for small RNAs. The results are expressed as values of the mean \pm standard deviation (SD). Data analysis was performed using the software StepOne, version 2.3 (Applied Biosystems). 


\section{Results}

\subsection{Strategy for Detecting Small RNAs of G. lamblia}

The most important consideration for designing a strategy to specifically detect a specific small RNA is to know its precise sequence. G. lamblia possesses snoRNAs, some of which have been proven to be precursors of mature miRNAs involved in the regulation of several genes [6,7]. We chose the snoRNA GlsR17 (ID: AY820298.1) and its derived miRNA (miR2) to design a stem-loop RT-qPCR-based strategy that could be routinely applied to detect and quantify small RNAs in G. lamblia.

The sequences corresponding to miR2 are located at the $3^{\prime}$-end of GlsR17 (Figure 1B) and a D box (CUAA) is present in the second hairpin structure of G. lamblia GlsR17, within the miR2 sequences, which identifies GlsR17 as a snoRNA of the C/D box class [46]. The relatively small size and the hairpin loop structures of GlsR17 qualify it as suitable substrate for Dicer action [5]. The sequence of the specific stem-loop-GlsR17 probe (based on the universal UPL21 probe) was designed with the software miRNA Primer Design Tool [32]. The stem-loop structure of the Stem-loop-GlsR17 probe hybridizing to the target small RNA is shown in Figure 1C; it contains the hexanucleotide GACTAT at the $3^{\prime}$-end, specific for capturing the GlsR17 and miR2 RNAs since they both possess the complementary AUAGUC sequence at their $3^{\prime}$-ends.

A)

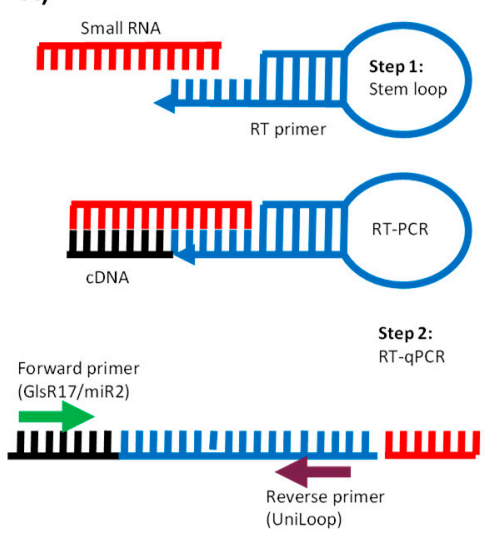

\section{孟典典典典监}

C)

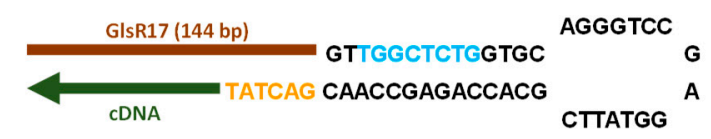

Universal ProbeLibrary probe \#21: Stem-loop:

GIsR17/miR2 specific sequence:
B)

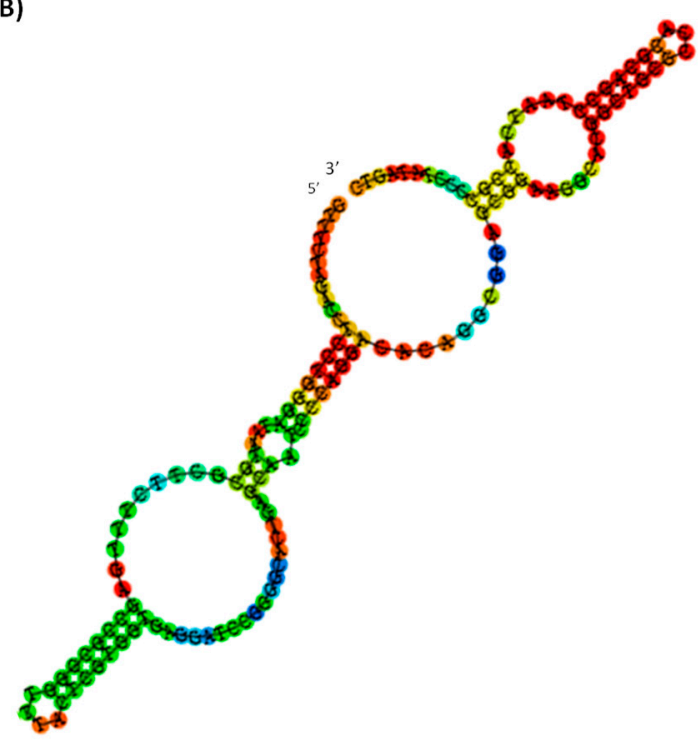

Figure 1. Stem-loop quantitative reverse transcription PCR (RT-qPCR) design to identify small RNAs of Giardia lamblia and schematic representation of the GlsR17 folding. (A) The scheme indicates the formation of complementary DNA (cDNA) from stem-loop-small RNA (step 1) and the quantification of the expression by RT-qPCR (step 2); (B) predicted structure of the small nucleolar RNA (snoRNA) GlsR17 based on RNAFold; (C) structure of the Stem-loop-GlsR17 probe hybridizing to the target small RNA; the cDNA generated by reverse transcription is shown in green color, the Universal ProbeLibrary (UPL) 21 region in blue, and the sequence specific for capturing the GlsR17 and miR2 RNAs (GACTAT) in yellow. 


\subsection{GlsR17 and miR2 Amplification by Stem-Loop RT-PCR}

The reported sizes for GlsR17 and miR2 are 144 and 25 nucleotides, respectively [6], so we used a sample of small RNA (see Materials and Methods) in addition to total RNA (Figure 2A) to perform the first-strand cDNA synthesis. According to the design of the primers for amplification by PCR, the expected sizes of the amplified fragments corresponding specifically to either GlsR17 or miR2 were $\approx 178$ and $61 \mathrm{bp}$, respectively. The agarose gel electrophoresis of the PCR-amplified products showed bands of the expected size for both GlsR17 and miR2 (Figure 2B). As can be observed, the gels showed no difference in size or intensity between the PCR products obtained from total RNA and from small RNA, which validates the use of the more readily obtainable total RNA for detection of small RNAs by stem-loop RT-PCR in G. lamblia. Because the resolutions of the agarose gels were not suitable, we decided to work with polyacrylamide gels for better definition of bands.

Then, the amplicons obtained for the GlsR17 by conventional PCR using a temperature gradient were resolved in these gels; no differences were found in products amplified between 60 and $75{ }^{\circ} \mathrm{C}$ from samples of total RNA or small RNAs (Figure 2C).

A)

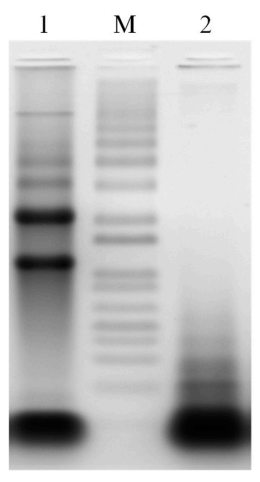

B)

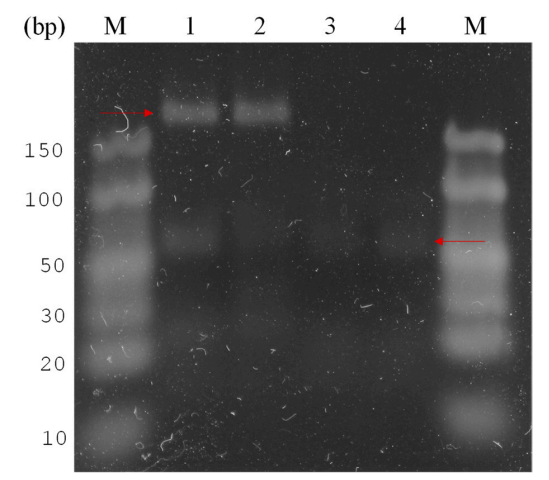

C)

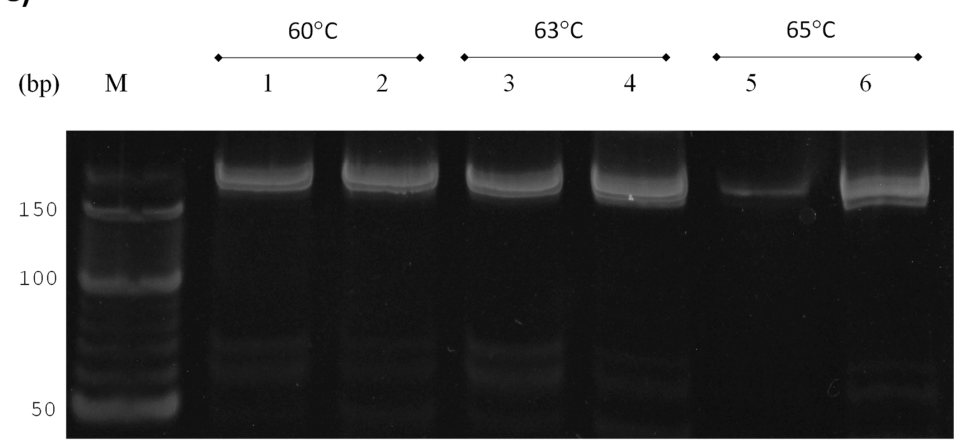

Figure 2. Identification of PCR-amplified fragments as GlsR17 and miR2 from G. lamblia. (A) Total RNA (lane 1) and small RNA (lane 3) extracted from G. lamblia trophozoites (see Materials and Methods) run on a $2 \%$ agarose gel; the size marker $(\mathrm{M})$ was $1 \mathrm{~kb}$ (lane 2) (O'GeneRuler $1 \mathrm{~kb}$ DNA Ladder, Thermo Scientific); (B) agarose gels (3\%) with the result of the amplification stem-loop RT-PCR analysis for GlsR17 and miR2 (lanes 1-4), respectively, using total RNA (lanes 1 and 3) or small RNA (lanes 2 and 4) as a template. The expected size for the GlsR17 amplified fragment was $178 \mathrm{bp}$, and for the miR2 fragment $61 \mathrm{bp}$, which is in accordance with the size of the bands appearing in the gels; (C) GlsR17 amplification from a temperature gradient by PCR and run on a $16 \%$ polyacrylamide gel. Products obtained from total RNA (lanes 1, 3 and 5) and small RNAs (lanes 2, 4 and 6). To determine the size of the fragments corresponding to the small RNAs the marker (M) O'RangeRuler $10 \mathrm{bp}$ DNA Ladder (Thermo Scientific) was used. 


\subsection{Amplification of miR2 by PCR}

Importantly, we demonstrated that from total RNA and small RNAs purified, both GlsR17 and miR2 were amplified. However, we needed to verify that the miRNA (miR2) obtained is not generated from the GlsR17 amplicon being used as template, and that it originated naturally by Dicer processing. For this reason, we decided to copurify small RNAs with sizes less than 100 bp (Figure 3A) from RNAs previously obtained (Figure 2A). When these small RNA fractions with less than 100 bp were used, amplicons of the snoRNA not were obtained despite using a temperature gradient between 60 and $64{ }^{\circ} \mathrm{C}$ (Figure 3B), while a $61 \mathrm{bp}$ amplicon was obtained with the primers corresponding to the miRNA miR2 (Figure 3B), and the miR2 products were obtained only at 63 and $64{ }^{\circ} \mathrm{C}$; this result led to the conclusion that the miR2 obtained was formed as a result of GlsR17 processing.

A)

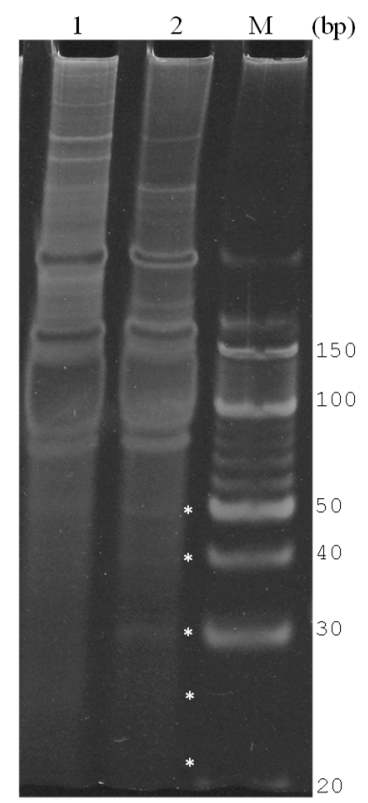

B)

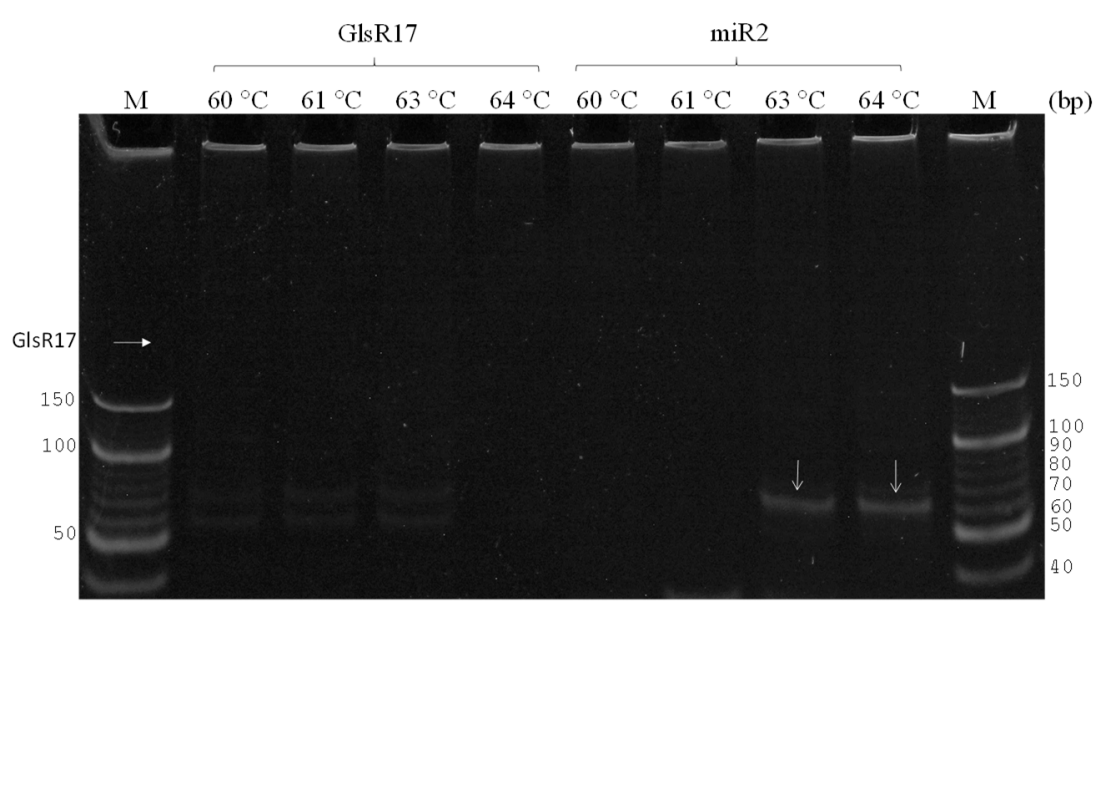

Figure 3. Size selection and gel extraction of small RNAs from total RNA and identification of PCR-amplified fragments of miR2. (A) Small RNAs obtained with sizes smaller than 100 bp copurified from total RNA (lane 1) and small RNA (lane 2), previously shown in Figure 2A. * Indicates bands of small RNAs between sizes 20 and $50 \mathrm{bp}$; (B) PCR was performed to verify the absence of GlsR17 and confirm the presence of miR2 in samples of small RNA less than $100 \mathrm{bp}$. Gradient of temperature was performed and the amplicons were observed on a $16 \%$ polyacrylamide gel.

After this, the amplified fragments were ligated to the pJET2.1/blunt vector and sequenced. The resulting sequences showed the presence of a $144 \mathrm{bp}$ fragment corresponding to GlsR17 (Figure 4) and the other sequence that corresponds to the cDNA synthesized from miR2, showing an identity of $100 \%$ with the sequence of AY820298 and L2708 reported in the NCBI BLASTN database. In both sequences, a D box sequence (CTAA) is present, which is a characteristic element of the snoRNA and miR2 of G. lamblia. 

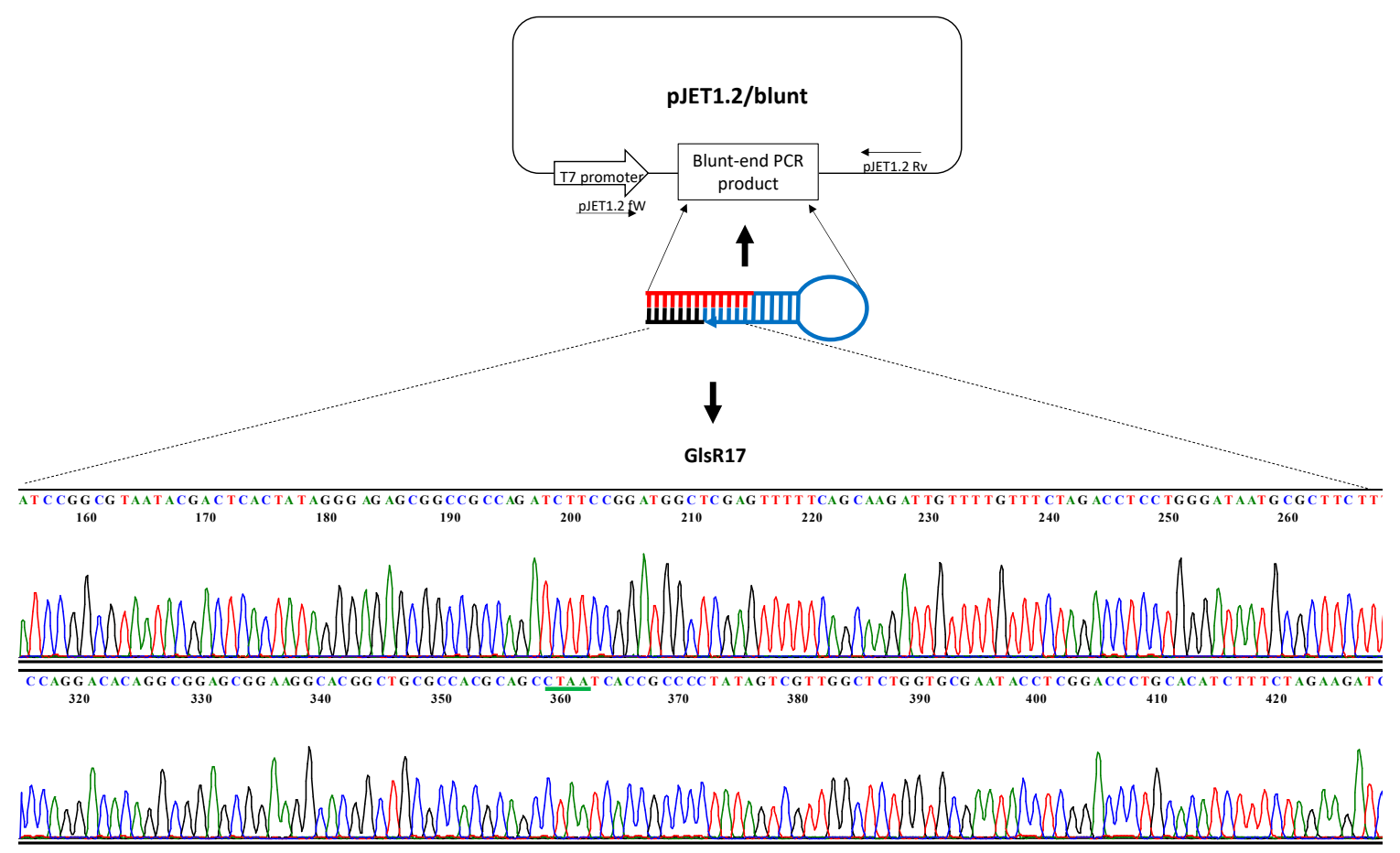

Figure 4. Identification of PCR-amplified fragments of GlsR17 from G. lamblia. PCR-amplified fragments with the primer pairs GlsR17-Fw and UniLoop were cloned into the pJET2.1/blunt vector and sequenced. The corresponding sequence identified the fragment of GlsR17. The green line indicates the sequences of the D box.

\subsection{Validation of Primers for $R T-q P C R$}

To set the conditions and validate the primers GlsR17-Fw, miR2-Fw, and UniLoop for RT-qPCR, we performed a series of PCR reactions using different amounts of cDNA, previously obtained with the probe Stem-loop-GlsR17, as a template (from 0.02 to $200 \mathrm{ng}$ ). As can be observed in Figure 5, amplification products of the expected size were obtained even with the smallest cDNA concentration for both GlsR17 (Figure 5A) and miR2 (Figure 5B). To test the specificity of the primers and confirm the results observed in the agarose gels, we performed RT-qPCR reactions in presence of SYBR Green and submitted the products to denaturation by increasing the temperature to obtain melt curves. A single well-defined sharp peak for each concentration was observed in both the GlsR17 and miR2 reactions (Figure 5C); the melting temperature $\left(\mathrm{T}_{\mathrm{m}}\right)$ for the amplified products, according to the melt curves, were $77.86^{\circ} \mathrm{C}$ and $83.37^{\circ} \mathrm{C}$, respectively. This result confirms that the oligonucleotides used in the reaction are specific for each of the two small RNAs. The amplification efficiency was $98 \%$ for GlsR17 and $101 \%$ for miR2, which are adequate for relative quantification of expression by RT-qPCR using the $2^{-\Delta \Delta \mathrm{Ct}}$ method $[42,43,45,47]$. 
A)

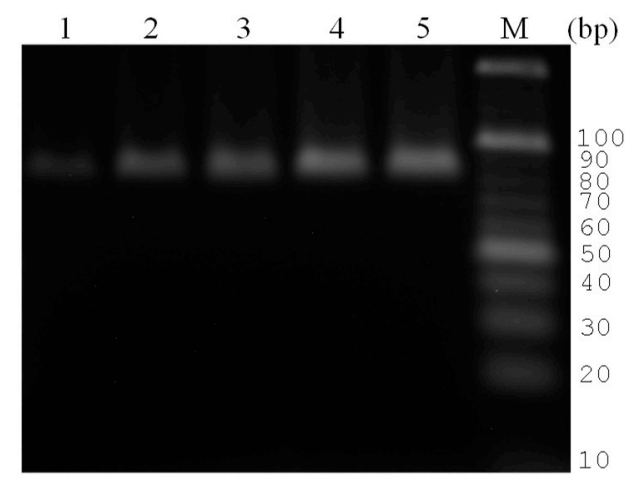

B)

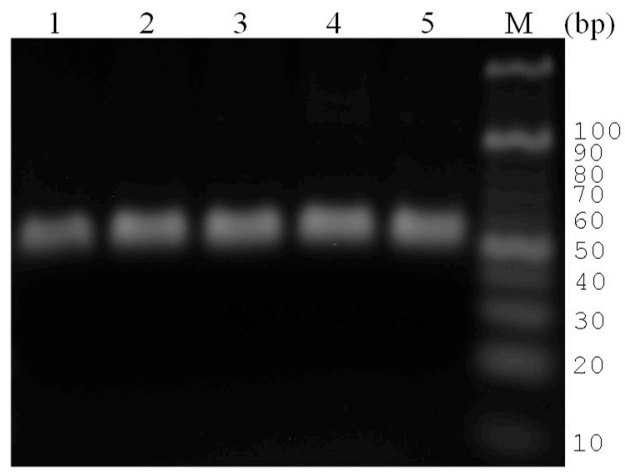

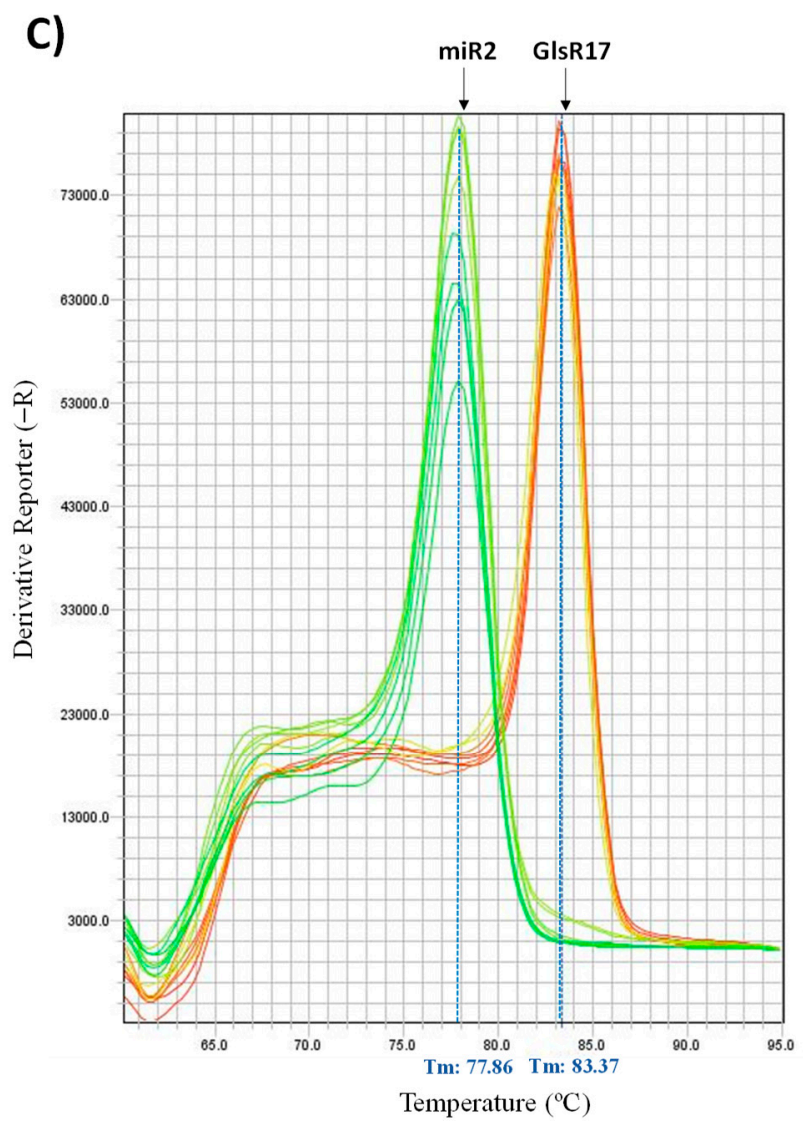

Figure 5. Amplification of the snoRNA GlsR17 and its derived miRNA (miR2) by PCR and RT-qPCR. Agarose gels ( $3 \%$ ) with the PCR amplification products obtained with primers GsIR17-Fw and UniLoop (A) and miR2-Fw and UniLoop (B) using different concentrations of template cDNA: lane 1: $0.025 \mathrm{ng}$, lane 2: $0.25 \mathrm{ng}$, lane 3: $2.5 \mathrm{ng}$, lane 4: $25 \mathrm{ng}$, lane 5: $250 \mathrm{ng}$. Lane M: O'RangeRuler ${ }^{\mathrm{TM}} 10 \mathrm{bp}$ DNA Ladder (Thermo Scientific). PCR conditions were: $95^{\circ} \mathrm{C}$ for $30 \mathrm{~s}$, and 40 cycles of $95^{\circ} \mathrm{C}$ for $3 \mathrm{~s}$ and $60{ }^{\circ} \mathrm{C}$ for $30 \mathrm{~s}$; (C) melt curves obtained for GlsR17 and miR2 amplification products by real-time PCR were generated using SYBR Green, and the same primers, template concentrations, and conditions as mentioned in A and B.

\subsection{Quantification of Expression of GlsR17 and miR2}

We performed an analysis of the abundance of GlsR17 and miR2 during the first $72 \mathrm{~h}$ of a culture of trophozoites in TYI-S-33 medium, with samples taken every $24 \mathrm{~h}$ (Figure 6). The analysis was performed by RT-qPCR according to the conditions established in the previous section, with $20 \mathrm{ng}$ of cDNA as a template. The results showed that from 24 to $72 \mathrm{~h}$ of culture, GlsR17 is present in similar abundance, with a slight increase from 24 to $48 \mathrm{~h}$, whereas miR2 abundance peaks at $48 \mathrm{~h}$ and drops to half the amount at $72 \mathrm{~h}$. The decrease of the miR2 expression could be mediated by changes of miRNAs expression in axenic culture, similar to the mRNA transcriptome changes of Giardia duodenalis trophozoites in these conditions for $96 \mathrm{~h}$ (analyzing stages of growth, stationary and declining), characterized by Ansell et al. [48]. Then, we consider that this change in the expression of miR2 is related to the expression of the transcriptome of G. lamblia in the time studied and with the regulation of different genes. Also, changes in the expression of VSP genes in Giardia have been reported, and could be related to the levels of the regulatory molecule, miR2. As previously demonstrated, miR2 is involved in regulating the expression of VSP genes, proteins that exhibit increase during stationary and decay stages $(60-96 \mathrm{~h})$ of G. lamblia trophozoite [6,48]. 


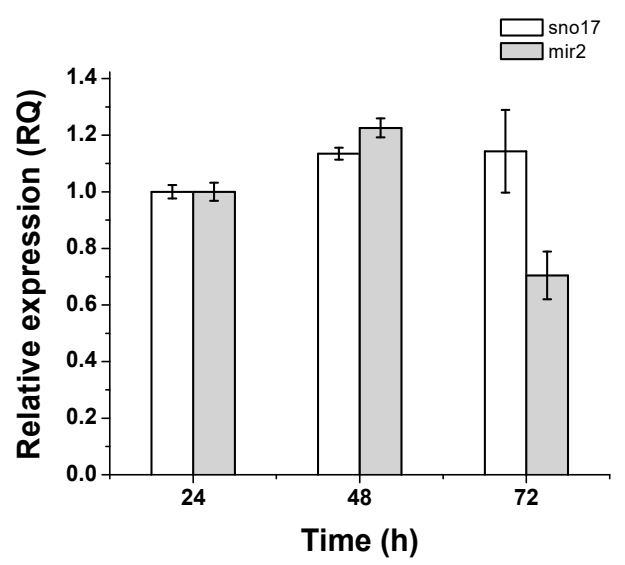

Figure 6. Quantification of the relative abundance of GlsR17 and miR2 from G. lamblia. The trophozoites were grown in TYI-S-33 medium for $72 \mathrm{~h}$ and the samples were taken every $24 \mathrm{~h}$. The error bars indicate standard deviation (SD) from five replicates, see Materials and Methods for details.

\subsection{Identification of miR2 Targets in VSP Genes}

The miRNA miR2 potentially participates in the regulation of at least 22 different genes encoding variant-specific surface proteins (VSPs), since putative miR2 binding sites are present at the $3^{\prime}$-untranslated regions ( $3^{\prime}$-UTR) of their mRNAs [6]. These authors also demonstrated the functionality of miR2 in translational repression of the reporter protein luciferase, expressed a genetically engineered gene that produces an mRNA containing miR2 binding sites at the $3^{\prime}$-UTR. Considering the importance of antigenic variation for successful G. lamblia infection and the role that miR2 seems to have in this phenomenon, we decided to perform a sequence alignment with a larger number of sequences of genes encoding VSP proteins. As shown in Figure 7, 33 VSP genes were found to have conserved sequences that may bind miR2.

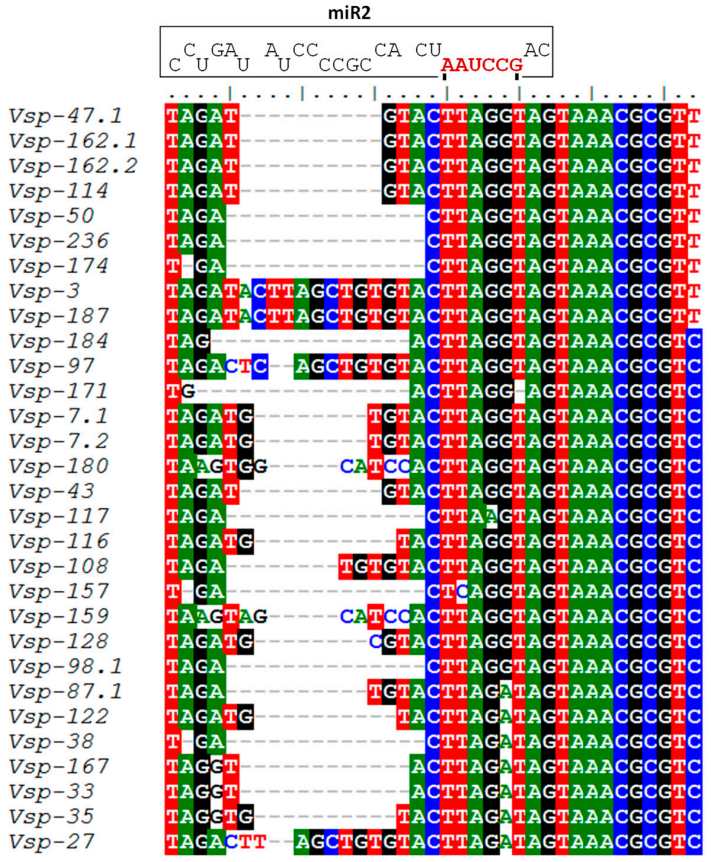

Figure 7. Alignment of different VSP genes. Conserved sequences and binding sites with the microRNA miR2 (brown color) in the $3^{\prime}$-untranslated region (3'-UTR) region of VSP genes from G. lamblia. VSP-47.1 (GL50803_117203), VSP-162.1 (GL50803_111936), VSP-162.2 (GL50803_111933), VSP-114 (GL50803_113304), VSP-50 (GL50803_137710), VSP-236 (GL50803_122564), VSP-174 (GL50803_137604), 
VSP-3 (GL50803_137740), VSP-187 (GL50803_89315), VSP-184 (GL50803_101380), VSP-97 (GL50803_10562), VSP-171 (GL50803_13402), VSP-7.1 (GL50803_136003), VSP-7.2 (GL50803_136004), VSP-180 (GL50803_137614), VSP-43 (GL50803_40591), VSP-117 (GL50803_97820), VSP-116 (GL50803_101765), VSP-108 (GL50803_101765), VSP-157 (GL50803_103916), VSP-159 (GL50803_112113), VSP-128 (GL50803_112207), VSP-98.1 (GL50803_112208), VSP-87.1 (GL50803_112647), VSP-122 (GL50803_113357), VSP-38 (GL50803_13194), VSP-167 (GL50803_134710), VSP-33 (GL50803_134711), VSP-25 (GL50803_37093), VSP-27 (GL50803_38901). The colors represent the following: red: thymine $(\mathrm{T})$; green: adenine $(\mathrm{A})$; black: guanine $(\mathrm{G})$; and blue: cytosine $(\mathrm{C})$. The alignment was made with the bioinformatic program BioEdit (Ibis Biosciences, Carlsbad, CA, USA), current version 7.2.5.

\section{Discussion}

The study of small RNAs, including miRNAs, siRNAs, and PIWI-interacting RNA (piRNA), is of great importance due to their role in gene regulation in different organisms [13,49]. One of the limitations in the study of these small molecules is the sensitivity of the methods used to detect a specific molecule and to analyze its expression levels in cells $[27,50,51]$. One method widely used for miRNAs detection and quantification is Northern blotting [24]; however, this methodology takes time for processing and may have low sensitivity [27]. Another method used has been primer extension [28]. It is thought that microarrays could improve the performance of the expression profiles of miRNAs, nonetheless, the method remains limited in terms of sensitivity (difficult to amplify the small RNA targets) and specificity (may lead to false positive signal) $[29,52,53]$. RT-qPCR has become the gold standard test for quantifying gene expression, however, applying it to miRNA or other small RNAs is a challenge since this technique requires a template that is at least twice as long as the specific forward or reverse primers, each typically $\approx 20 \mathrm{nt}$ in length. Consequently, the target minimum length has to be around $40 \mathrm{bp}$, which makes most mature miRNAs and siRNAs too short for detection and quantification by standard RT-qPCR methods [30,53,54]. Another technique that could be adapted for the identification of small RNA is loop-mediated isothermal amplification (LAMP), which is a novel nucleic acid amplification method. Amplification and detection of amplicons can be completed in a single step, by incubating all the reagents in a single tube [55]. However, the LAMP reaction requires a set specially designed primers, uses four (or six) primers targeting six (or eight) regions with significant restrictions imposed on their respective positioning and orientation $[55,56]$. Another limitation for this technique, as well as those previously mentioned, is that they are not useful for cloning, and, therefore, it is not possible to get the sequence of the obtained amplicons.

Therefore, it is of utmost interest to have techniques available that can reliably detect and quantify small RNAs. In this sense, the stem-loop RT-qPCR technique has proven to be highly specific to detect and suitable to quantify specific small RNAs [31-33,36,53,57]. Moreover, this technique is fast and easy to perform and has a lower cost compared with Northern blotting. In this work, we have established the conditions for routine analysis of small RNAs in Giardia: the RNA extraction method, the design of the stem-loop probe and PCR primers, and the conditions of RT-qPCR to quantify the expression of specific small RNAs, using the snoRNA GlsR17 and its derived miRNA product (miR2) as isolation and quantification tests.

snoRNAs are essential for the maturation of ribosomal RNA, and are localized to the nucleolus $[5,46]$. Some snoRNAs also serve as precursors for the biogenesis of mature miRNAs that participate in gene regulation $[5,9,10,58]$. This is the case for some snoRNAs of G. lamblia [6,7]. Previous studies of miRNAs in G. lamblia were performed by small RNA isolation and ligation to RNA linkers [6], construction of a small RNA library in a plasmid vector [17], and bioinformatic sequence analysis followed by primer extension [7]. The miRNA miR2 was identified as a Dicer-processed product of the snoRNA GlsR17 and localized to the cytoplasm by FISH, whereas GlsR17 was found primarily in the nucleolus of only one of the two nuclei in Giardia [6]. The feasibility to detect and 
quantify specific miRNAs and other small regulatory RNAs by stem-loop RT-qPCR in G. lamblia will greatly contribute to the study of gene regulation and the role of miRNA in the possible antigenic variation in this parasite.

G. lamblia infections are associated with antigenic variation, which is generated by a continuous change of the VSPs expressed. From a repertory of around 190 VSP-encoding genes, Giardia can express only one VSP on the surface of each parasite at a particular time, and it spontaneously changes to a different VSP by unknown mechanisms [21]. The differential expression of GlsR17 and miR2 that we found in this study may be related to the timing of expression of VSP genes [48]. Recently, an analysis performed on the transcriptome of G. duodenalis showed transcriptional variation in axenic culture, during 96 hours, which includes the log, stationary, and declining stages of the in vitro culture [48]. As with this differential gene expression, miRNAs may also change their expression, as was observed with miR2 in this study (Figure 6). Although, we now observe that VSP-116 (GL50803_101765) is one of the genes expressed mostly after $60 \mathrm{~h}$ of culture, and according to the alignment performed for these genes and their miR2 binding sites, this gene or others could be regulated by this miRNA (Figure 7). In addition, the increase in VSP-116 gene expression may be due to the fact that miR2 negatively regulates its expression, among other genes, and these changes may be necessary for the survival of G. lamblia. This possible regulation by microRNA in G. lamblia, from which a bioinformatic analysis was conducted predicting different microRNAs derived from snoRNAs, and putative target sites were identified at the $3^{\prime}$-UTR of variant surface protein mRNAs.

The results reported by these authors confirmed that each of the miRNAs represses the translation of a reporter (luciferase gene) transcript tagged with their respective target sites, and this repression requires the presence of the Ago protein from Giardia, suggesting that it is supported by an RNA-induced silencing complex (RISC)-mediated mechanism [6,17,18].

\section{Conclusions}

In summary, our results demonstrate the feasibility of using the stem-loop RT-qPCR method for routinely and efficiently detecting and quantifying specific small RNAs (snoRNA/dsRNA/siRNA/miRNA) from total RNA in G. lamblia. Interestingly, we only used three primers to identify two small RNAs, one of them with a stem-loop to capture both small RNAs (GlsRNA17 and miR2), another that helped to obtain a specific small RNA by RT-PCR, and a third primer present in the internal sequence of the stem-loop (UniLoop-rev), which served to confirm by conventional PCR the capture of some small RNA. This will contribute to the study of gene regulation in G. lamblia, and in particular, to the role of miRNAs in antigenic variation through silencing of the expression of VSP proteins.

Acknowledgments: Jaime Marcial-Quino is supported by CONACYT grant 259201 and Cátedras CONACYT (2184) project number 2057. Saúl Gómez-Manzo is supported by CONACyT grant 154570 and INP 055/2015; Edgar Sierra-Palacios is supported by the Universidad Autónoma de la Ciudad de México with the agreement (UACM/SECITI/060/2013, project PI2014-28). The technical assistance of Carmen Ortiz is greatly appreciated. Thank you also to Javier Gallegos Infante (Instituto de Fisiología Celular, UNAM) for assistance with bibliographic materials.

Author Contributions: Jaime Marcial-Quino, Saúl Gómez-Manzo, Francisco Fierro and Edgar Sierra-Palacios conceived and designed the experiments. Jaime Marcial-Quino, America Vanoye-Carlo, Yadira Rufino-Gonzalez, Adriana Castillo-Villanueva, Rosa Angélica Castillo-Rodríguez and Horacio Reyes-Vivas performed the experiments. Jaime Marcial-Quino, Saúl Gómez-Manzo, Francisco-Fierro, Eduardo Rodríguez-Bustamante and Roberto Arreguin-Espinosa analyzed the data. All authors wrote the manuscript.

Conflicts of Interest: The authors declare no conflict of interest.

\section{References}

1. Lebwohl, B.; Deckelbaum, R.J.; Green, P.H.R. Giardiasis. Gastrointest. Endosc. 2003, 57, 906-913. [CrossRef]

2. Müller, N.; von Allmen, N. Recent insights into the mucosal reactions associated with Giardia lamblia infections. Int. J. Parasitol. 2005, 35, 1339-1347. [CrossRef] [PubMed] 
3. Plutzer, J.; Ongerth, J.; Karanis, P. Giardia taxonomy, phylogeny and epidemiology: Facts and open questions. Int. J. Hyg. Environ. Health 2010, 213, 321-333. [CrossRef] [PubMed]

4. Zheng, Y.; Cai, X.; Bradley, J.E. microRNAs in parasites and parasite infection. RNA Biol. 2013, 10, 371-379. [CrossRef] [PubMed]

5. Scott, M.S.; Ono, M. From snoRNA to miRNA: Dual function regulatory non-coding RNAs. Biochimie 2011, 93, 1987-1992. [CrossRef] [PubMed]

6. Saraiya, A.A.; Wang, C.C. snoRNA, a novel precursor of microRNA in Giardia lamblia. PLoS Pathog. 2008, 4, e1000224. [CrossRef] [PubMed]

7. Li, W.; Saraiya, A.A.; Wang, C.C. The profile of snoRNA-derived microRNAs that regulate expression of variant surface proteins in Giardia lamblia. Cell. Microbiol. 2012, 14, 1455-1473. [CrossRef] [PubMed]

8. Kawaji, H.; Hayashizaki, Y. Exploration of Small RNAs. PLoS Genet. 2008, 4, e22. [CrossRef] [PubMed]

9. Bratkovič, T.; Rogelj, B. The many faces of small nucleolar RNAs. Biochim. Biophys. Acta 2014, 1839, 438-443. [CrossRef] [PubMed]

10. Eliaz, D.; Doniger, T.; Tkacz, I.D.; Biswas, V.K.; Gupta, S.K.; Kolev, N.G.; Unger, R.; Ullu, E.; Tschudi, C.; Michaeli, S. Genome-wide analysis of small nucleolar RNAs of Leishmania major reveals a rich repertoire of RNAs involved in modification and processing of rRNA. RNA Biol. 2015, 12, 1222-1255. [CrossRef] [PubMed]

11. Kolev, N.G.; Tschudi, C.; Ullu, E. RNA interference in protozoan parasites: Achievements and challenges. Eukariot. Cell 2011, 10, 1156-1163. [CrossRef] [PubMed]

12. Shabalina, S.A.; Koonin, E.V. Origins and evolution of eukaryotic RNA interference. Trends Ecol. Evol. 2008, 23, 578-587. [CrossRef] [PubMed]

13. Bartel, D.P. MicroRNAs: Genomics, biogenesis, mechanism, and function. Cell 2004, 116, 281-297. [CrossRef]

14. Davis-Dusenbery, B.N.; Hata, A. Mechanisms of control of microRNA biogenesis. J. Biochem. 2010, 48, 381-392.

15. Treiber, T.; Treiber, N.; Meister, G. Regulation of microRNA biogenesis and function. Thromb. Haemost. 2012, 107, 605-610. [CrossRef] [PubMed]

16. Ha, M.; Kim, V.N. Regulation of microRNA biogenesis. Nat. Rev. Mol. Cell Biol. 2014, 15, 509-524. [CrossRef] [PubMed]

17. Saraiya, A.A.; Li, W.; Wang, C.C. A microRNA derived from an apparent canonical biogenesis pathway regulates variant surface protein gene expression in Giardia lamblia. RNA 2011, 17, 2152-2164. [CrossRef] [PubMed]

18. Saraiya, A.A.; Li, W.; Wu, J.; Chang, C.H.; Wang, C.C. The microRNAs in an ancient protist repress the variant-specific surface protein expression by targeting the entire coding sequence. PLoS Pathog. 2014, 10, e1003791. [CrossRef] [PubMed]

19. Guo, J.; Zheng, W.; Wang, Y.; Li, Y.; Lu, S.; Feng, X. Coexistence of sense and anti-sense mRNAs of variant surface protein in Giardia lamblia trophozoites. Biochem. Biophys. Res. Commun. 2014, 444, 439-444. [CrossRef] [PubMed]

20. Watanabe, T.; Totoki, Y.; Toyoda, A.; Kaneda, M.; Kuramochi-Miyagawa, S.; Obata, Y.; Chiba, H.; Kohara, Y.; Kono, T.; Nakano, T.; et al. Endogenous siRNAs from naturally formed dsRNAs regulate transcripts in mouse oocytes. Nature 2008, 453, 539-543. [CrossRef] [PubMed]

21. Prucca, C.G.; Slavin, I.; Quiroga, R.; Elías, E.V.; Rivero, F.D.; Saura, A.; Carranza, P.G.; Luján, H.D. Antigenic variation in Giardia lamblia is regulated by RNA interference. Nature 2008, 456, 750-754. [CrossRef] [PubMed]

22. Prucca, C.G.; Rivero, F.D.; Luján, H.D. Regulation of antigenic variation in Giardia lamblia. Annu. Rev. Microbiol. 2011, 65, 611-630. [CrossRef] [PubMed]

23. Liao, J.Y.; Guo, Y.H.; Zheng, L.L.; Li, Y.; Xu, W.L.; Zhang, Y.C.; Zhou, H.; Lun, Z.R.; Ayala, F.J.; Qu, L.H. Both endo-siRNAs and tRNA-derived small RNAs are involved in the differentiation of primitive eukaryote Giardia lamblia. Proc. Natl. Acad. Sci. USA 2014, 111, 14159-14164. [CrossRef] [PubMed]

24. Kolev, N.G.; Ullu, E. snoRNAs in Giardia lamblia: A novel role in RNA silencing? Trends Parasitol. 2009, 25, 348-350. [CrossRef] [PubMed]

25. Lau, N.C.; Lim, L.P.; Weinstein, E.G.; Bartel, D.P. An abundant class of tiny RNAs with probable regulatory roles in Caenorhabditis elegans. Science 2001, 294, 858-862. [CrossRef] [PubMed]

26. Várallyay, E.; Burgyán, J.; Havelda, Z. MicroRNA detection by northern blotting using locked nucleic acid probes. Nat. Protoc. 2008, 3, 190-196. 
27. Cissell, K.A.; Deo, S.K. Trends in microRNA detection. Anal. Bioanal. Chem. 2009, 394, 1109-1116. [CrossRef] [PubMed]

28. Zeng, Y.; Cullen, B.R. Sequence requirements for micro RNA processing and function in human cells. RNA 2003, 9, 112-123. [CrossRef] [PubMed]

29. Li, W.; Ruan, K. MicroRNA detection by microarray. Anal. Bioanal. Chem. 2009, 394, 1117-1124. [CrossRef] [PubMed]

30. Cirera, S.; Busk, P.K. Quantification of miRNAs by a simple and specific qPCR method. Methods Mol. Biol. 2014, 1182, 73-81.

31. Kramer, M.F. Stem-loop RT-qPCR for miRNAS. Curr. Protoc. Mol. Biol. 2011. [CrossRef]

32. Czimmerer, Z.; Hulvely, J.; Simandi, Z.; Varallyay, E.; Havelda, Z.; Szabo, E.; Varga, A.; Dezso, B.; Balogh, M.; Horvath, A.; et al. A Versatile Method to Design Stem-Loop Primer-Based Quantitative PCR Assays for Detecting Small Regulatory RNA Molecules. PLoS ONE 2013, 8, e55168. [CrossRef] [PubMed]

33. Yang, L.H.; Wang, S.L.; Tang, L.L.; Liu, B.; Ye, W.L.; Wang, L.L.; Wang, Z.Y.; Zhou, M.T.; Chen, B.C. Universal Stem-Loop Primer Method for Screening and Quantification of MicroRNA. PLoS ONE 2014, 9, e115293. [CrossRef] [PubMed]

34. Gautam, V.; Singh, A.; Singh, S.; Sarkar, A.K. An Efficient LCM-Based Method for Tissue Specific Expression Analysis of Genes and miRNAs. Sci. Rep. 2016, 6, 21577. [CrossRef] [PubMed]

35. Balcells, I.; Cirera, S.; Busk, P.K. Specific and sensitive quantitative RT-PCR of miRNAs with DNA primers. BMC Biotechnol. 2011, 11, 70. [CrossRef] [PubMed]

36. Busk, P.K. A tool for design of primers for microRNA-specific quantitative RT-qPCR. BMC Bioinform. 2014, 15, 29. [CrossRef] [PubMed]

37. Malone, C.; Brennecke, J.; Czech, B.; Aravin, A.; Hannon, G.J. Preparation of Small RNA Libraries for High-Throughput Sequencing. Cold Spring Harb. Protoc. 2012, 2012, 1067-1077. [CrossRef] [PubMed]

38. Nielsen, T.W. Gel Purification of RNA. Cold Spring Harb. Protoc. 2013, 180-183. [CrossRef] [PubMed]

39. Aurrecoechea, C.; Brestelli, J.; Brunk, B.P.; Carlton, J.M.; Dommer, J.; Fischer, S.; Gajria, B.; Gao, X.; Gingle, A.; Grant, G.; et al. GiardiaDB and TrichDB: Integrated genomic resources for the eukaryotic protist pathogens Giardia lamblia and Trichomonas vaginalis. Nucleic Acids Res. 2009, 37, D526-D530. [CrossRef] [PubMed]

40. Martínez, I.; Nogueda, B.; Martínez-Hernández, F.; Espinoza, B. Microsatellite and mini-exon analysis of Mexican human DTU I Trypanosoma cruzi strains and their susceptibility to nifurtimox and benznidazole. Vector Borne Zoonotic Dis. 2013, 13, 181-187. [CrossRef] [PubMed]

41. Ramakers, C.; Ruijter, J.M.; Deprez, R.H.; Moorman, A.F. Assumption-free analysis of quantitative real-time polymerase chain reaction (PCR) data. Neurosci. Lett. 2003, 339, 62-66. [CrossRef]

42. Wilkening, S.; Bader, A. Quantitative real-time polymerase chain reaction: Methodical analysis and mathematical model. J. Biomol. Tech. 2004, 15, 107-111. [PubMed]

43. Pfaffl, M.W. Quantification Strategies in Real-Time PCR. In The Real-Time PCR Encyclopedia, A-Z of Quantitative PCR; Bustin, S.A., Ed.; International University Line: La Jolla, CA, USA, 2004; pp. 87-120.

44. Livak, K.J.; Schmittgen, T.D. Analysis of relative gene expression data using real-time quantitative PCR and the $2^{-\Delta \Delta \mathrm{Ct}}$ method. Methods 2001, 25, 402-408. [CrossRef] [PubMed]

45. Marcial-Quino, J.; Fierro, F.; De la Mora-De la Mora, I.; Enríquez-Flores, S.; Gómez-Manzo, S.; Vanoye-Carlo, A.; Garcia-Torres, I.; Sierra-Palacios, E.; Reyes-Vivas, H. Validation of housekeeping genes as an internal control for gene expression studies in Giardia lamblia using quantitative real-time PCR. Gene 2016, 581, 21-30. [CrossRef] [PubMed]

46. Reichow, S.L.; Hamma, T.; Ferre-D'Amare, A.R.; Varani, G. The structure and function of small nucleolar ribonucleoproteins. Nucleic Acids Res. 2007, 35, 1452-1464. [CrossRef] [PubMed]

47. Fernández-Aparicio, M.; Huang, K.; Wafula, E.K.; Honaas, L.A.; Wickett, N.J.; Timko, M.P.; dePamphilis, C.W.; Yoder, J.; Westwood, J.H. Application of qRT-PCR and RNA-Seq analysis for the identification of housekeeping genes useful for normalization of gene expression values during Striga hermonthica development. Mol. Biol. Rep. 2013, 40, 3395-3407. [CrossRef] [PubMed]

48. Ansell, B.R.E.; McConville, M.J.; Baker, L.; Korhonen, P.K.; Young, N.D.; Hall, R.S.; Rojas, C.A.A.; Svärd, S.G.; Gasser, R.B.; Jex, A.R. Time-Dependent Transcriptional Changes in Axenic Giardia duodenalis Trophozoites. PLoS Negl. Trop. Dis. 2015, 9, e0004261. [CrossRef] [PubMed]

49. Thomson, T.; Lin, H. The biogenesis and function of PIWI proteins and piRNAs: Progress and prospect. Annu. Rev. Cell Dev. Biol. 2009, 25, 355-376. [CrossRef] [PubMed] 
50. Tian, T.; Wang, J.; Zhou, X. A review: microRNA detection methods. Org. Biomol. Chem. 2015, 13, $2226-2238$. [CrossRef] [PubMed]

51. Krichevsky, A.M.; King, K.S.; Donahue, C.P.; Khrapko, K.; Kosik, K.S. A microRNA array reveals extensive regulation of microRNAs during brain development. RNA 2003, 9, 1274-1281. [CrossRef] [PubMed]

52. Liu, C.G.; Calin, G.A.; Meloon, B.; Gamliel, N.; Sevignani, C.; Ferracin, M.; Dumitru, C.D.; Shimizu, M.; Zupo, S.; Dono, M.; et al. An oligonucleotide microchip for genome-wide microRNA profiling in human and mouse tissues. Proc. Natl. Acad. Sci. USA 2004, 101, 9740-9744. [CrossRef] [PubMed]

53. Chen, C.; Ridzon, D.A.; Broomer, A.J.; Zhou, Z.; Lee, D.H.; Nguyen, J.T.; Barbisin, M.; Xu, N.L.; Mahuvakar, V.R.; Andersen, M.R.; et al. Real-time quantification of microRNAs by stem-loop RT-PCR. Nucleic Acids Res. 2005, 33, e179. [CrossRef] [PubMed]

54. Chen, C.; Tan, R.; Wong, L.; Fekete, R.; Halsey, J. Quantitation of microRNAs by real-time RT-qPCR. Methods Mol. Biol. 2011, 687, 113-134.

55. Parida, M.; Sannarangaiah, S.; Dash, P.K.; Rao, P.V.; Morita, K. Loop mediated isothermal amplification (LAMP): A new generation of innovative gene amplification technique; perspectives in clinical diagnosis of infectious diseases. Rev. Med. Virol. 2008, 18, 407-421. [CrossRef] [PubMed]

56. Gandelma, O.; Jackson, R.; Kiddle, G.; Tisi, L. Loop-Mediated Amplification Accelerated by Stem Primers. Int. J. Mol. Sci. 2011, 12, 9108-9124. [CrossRef] [PubMed]

57. Bissels, U.; Wild, S.; Tomiuk, S.; Holste, A.; Hafner, M.; Tuschl, T.; Bosio, A. Absolute quantification of microRNAs by using a universal reference. RNA 2009, 15, 2375-2384. [CrossRef] [PubMed]

58. Lin, W.C.; Li, S.C.; Lin, W.C.; Shin, J.W.; Hu, S.N.; Yu, X.M.; Huang, T.Y.; Chen, S.C.; Chen, H.C.; Chen, S.J.; et al. Identification of microRNA in the protist Trichomonas vaginalis. Genomics 2009, 93, 487-493. [CrossRef] [PubMed]

(C) 2016 by the authors; licensee MDPI, Basel, Switzerland. This article is an open access article distributed under the terms and conditions of the Creative Commons Attribution (CC-BY) license (http://creativecommons.org/licenses/by/4.0/). 